\title{
Supportive Periodontal Therapy- An Important Landmark in Clinical Practice
}

\author{
Amit Lakhani ${ }^{1}$, Ena Sharma², Supreet K ${ }^{3}$, Surinder Sachdeva ${ }^{4}$, Savita Kapila ${ }^{5}$ \\ ${ }^{1}$ Department of Orthopaedics, Maharishi Markandeshwar Medical College \& Hospital, Mullana, Ambala, \\ Haryana, India. ${ }^{2}$ Department of Periodontics, Maharishi Markandeshwar College of Dental Sciences and \\ Research, Mullana, Ambala Haryana, India. ${ }^{3}$ Department of Periodontics, Maharishi Markandeshwar \\ Institute of Medical Sciences, Mullana, Ambala, Haryana, India. ${ }^{4}$ Department of Periodontics, Maharishi \\ Markandeshwar Institute of Medical Sciences, Mullana, Ambala, Haryana, India. ${ }^{5}$ Department of \\ Periodontics, Maharishi Markandeshwar Institute of Medical Sciences \& Research, Mullana, Ambala, \\ Haryana, India.
}

\section{INTRODUCTION}

The long term success of periodontal therapy depends less on the manner in which the case was treated than on vigorous follow-up of the wound healing process immediately after therapy and on how well the case is maintained in subsequent recall (Rosling et al 1976, Nyman et al 1977, Knowles et al 1979, Ramfjord et al 1982, West felt et al 1985) SPT has gone by many names, including recall and maintenance, but the name was changed at the 1989 World Workshop in Clinical Periodontics to reflect the fact that the long-term treatment provided for patients during supportive periodontal treatment is of critical importance to the survival of the dentition. In most cases, this form of therapy is used following the completion of active periodontal therapy, but it can be used in other phases of treatment as well. ${ }^{1}$

SPT is an absolute prerequisite to guarantee beneficial treatment outcomes with maintained levels of clinical attachment over long periods of time. While the maintenance of treatment results for the majority of patients has been documented up to 14 years, it has to be realized that a small proportion of patients will experience recurrent infections with progression of periodontal lesions in a few sites in a completely unpredictable mode. A series of longitudinal studies on periodontal therapeutic modalities was performed in the past 25 years, first at the University of Michigan, later at the University of Gothenburg, Sweden, and also at the Universities of Minnesota, Nebraska and Loma Linda. ${ }^{2}$ These studies always incorporated the patients into a well-organized maintenance care system with recall visits at regular intervals (generally 3-4 months). Although the patients performed plaque control with various degrees of efficacy, the SPT resulted in excellent maintenance of postoperative attachment levels in most patients (Knowles 1973, Ramfjord et al. 1982). ${ }^{3}$

The rationale for 3 months recall intervals for SPT is most likely based on published studies that used 3-4 month intervals as part of study design rather than a result of studies comparing the efficacy and safety of different time intervals for SPT. Another rationale for short intervals between clinic visits is the understanding that frequent maintenance care is necessary to eliminate/reduce subgingival proportions of pathogens associated with periodontitis. Recolonisation of pathogens in previously treated periodontal pockets occurs quickly if oral hygiene is not properly maintained. Therefore, 3-4-month maintenance care intervals have been suggested. However, several other studies have demonstrated that longer intervals between maintenance care visits can effectively prevent further disease progression. ${ }^{4}$

\author{
Corresponding Author: \\ Dr. Ena Sharma, \\ Maharishi Markandeshwar College and \\ Dental Sciences and Research, Mullana, \\ Ambala, Haryana, India. \\ E-mail:dr.sharma_ena@yahoo.co.in
}

DOI: $10.14260 / j e m d s / 2020 / 175$

Financial or Other Competing Interests: None.

How to Cite This Article:

Lakhani A, Sharma E, Supreet $K$, et al. Supportive periodontal therapy- an important landmark in clinical practice. J. Evolution Med. Dent. Sci. 2020;9(10):810813, DOI: 10.14260/jemds/2020/175

Submission 16-11-2019,

Peer Review 18-02-2020,

Acceptance 24-02-2020,

Published 09-03-2020.

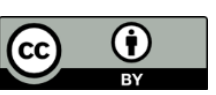


Axelsson et al., in a 15-year follow up study of 375 adult individuals, demonstrated a low incidence of caries and almost no further loss of periodontal support even though maintenance visits were performed only once or twice yearly for the previous 9 years. ${ }^{5}$ Lindhe et al., using a maintenance program restricted to oral hygiene instruction and supragingival cleaning every 4-6 months, found that patients who consistently had a high frequency of plaque-free surfaces showed little evidence of additional loss of attachment. Thus rigorous oral hygiene, frequent recalls do not appear to be as important as in individuals with inadequate oral hygiene Lovdal et al., 1961 in a 5 year observation of 1428 adults from an industrial company in Oslo with a recall period 2-4 times/year. (scaling and root planing + oral hygiene instructions given) showed an improvement of gingival condition by $60 \%$ and reduction in tooth loss by $50 \%$ in all the patients. ${ }^{5}$

The goal of periodontal treatment is to maintain the natural dentition in functional health and comfort throughout the lifetime. This ideal goal often is not completely met in most of the cases, because it requires perfect plaque control, which is seldom achieved. One likely explanation for the recurrence of periodontal disease is incomplete subgingival plaque removal which causes reestablishment of subgingival microflora. Supportive periodontal treatment is an integral part of periodontal therapy. Procedures are performed at selected intervals to assist the periodontal patients in maintaining oral health. The main objective of SPT is to support the results of the initial therapy through a periodic professional recall system and maintenance of optimal plaque control, supragingivally and subgingivally. However, in clinical practice there is an overwhelming risk that neither the initial treatment nor the subsequent personal oral hygiene will be perfect for every tooth for every treated periodontitis patient. Thus, SPT is indicated for every periodontitis patient with significant (mm or more) loss of attachment. This case report emphasizes the importance of supportive periodontal therapy in the maintenance of periodontal health on a long term basis.

\section{Therapeutic Objectives: ${ }^{1}$}

1. To prevent the progression and recurrence of periodontal disease in patient who have previously been treated for gingivitis and Periodontitis;

2. To prevent the loss of dental implants after clinical stability has been achieved;

3. To reduce tooth loss by monitoring the dentition and any prosthetic replacements of the natural teeth;

4. To diagnose and manage, in a timely manner other disease or condition found which is related to the oral cavity.

\section{PRESENTATION OF CASE}

A 36 years old male reported to the department 10 months back, complaining of loose teeth since 5 years, sensitivity of teeth from 8 months. He gave a history of full mouth flap surgeries being done 5 years back which had thus improved his dental condition for a certain period after which the periodontal status began to deteriorate and had attained present status. The teeth had gradually become loose, the sensitivity of teeth aggravated on taking cold foods and subsided on its own. There was no relevant medical and family history, No abnormalities were seen on extra oral examination. (figure 1,2)
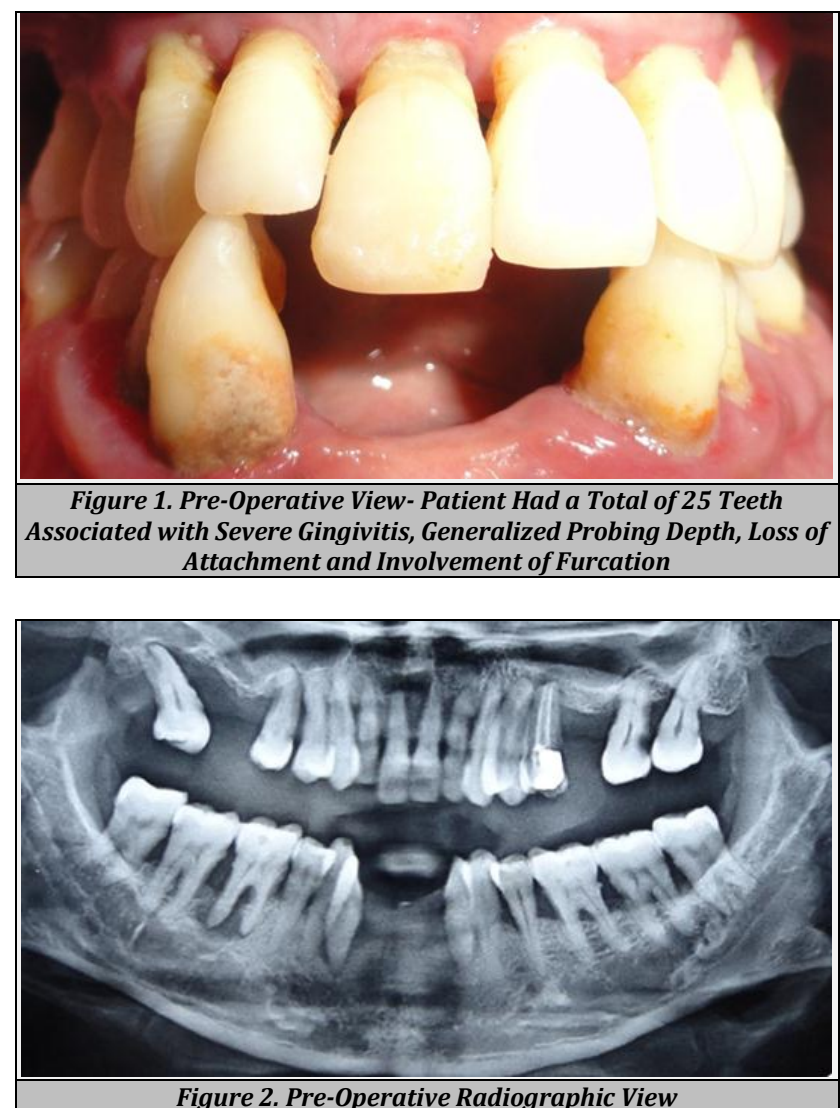

CLINICAL DIAGNOSIS

Patient was diagnosed with generalized aggressive periodontitis with overall unfavourable prognosis. Maxillary teeth had hopeless prognosis. 43 and 33 also had hopeless prognosis, whereas 46 was involved periapically. So, a questionable prognosis was given. All the other remaining teeth had fair prognosis.

\section{DISCUSSION OF MANAGEMENT}

The teeth with hopeless prognosis were advised for extraction. Patient was motivated; oral hygiene instructions were given and re-instated at every visit. Phase I therapy, which includes scaling and root planing (SRP) was done. The patient was rescheduled for periodontal surgery after 4 weeks. 46 was treated endodontically. Access flap surgery IRT 34-38 and 4448 was done. Patient was on a recalled visit every month for check-up.

\section{DISCUSSION}

SPT is an integral part of the periodontal therapy which could be based on Merin's classification. SPT should include an update of medical and dental histories, radiographs, extra oral 
and intraoral soft tissue examination, dental examination, periodontal evaluation, removal of bacterial plaque from the supragingival and subgingival regions, scaling and root planning where indicated and a review of patient's plaque control efficiency and other appropriate behaviour modification. ${ }^{1,6}$

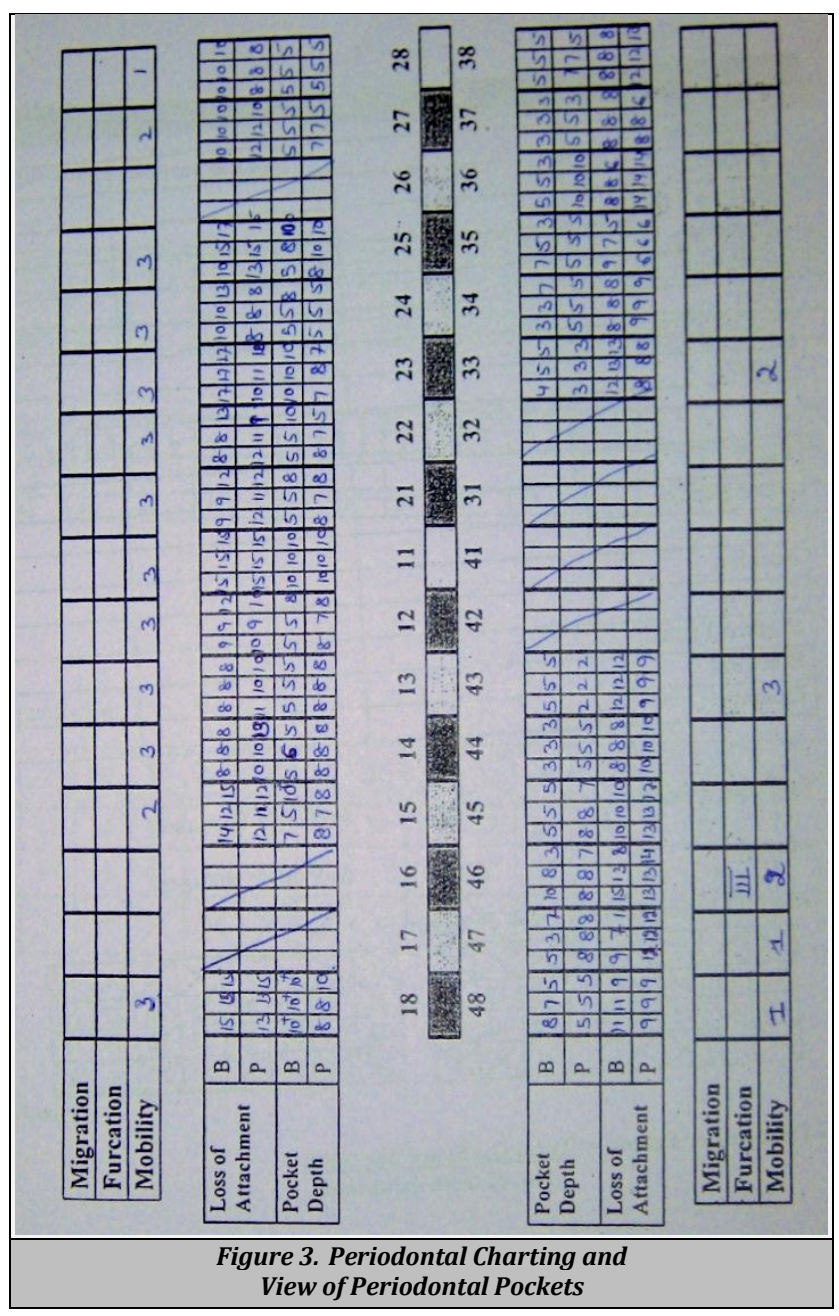

\section{Classification of Periodontal Maintenance Therapy 7}

- $\quad$ Preventive SPT, designed to prevent the inception of disease in individuals without periodontal pathosis.

- Trial SPT, designed to maintain border line periodontal conditions over a period to further assess the need for corrective therapy for such problems as inadequate gingiva, gingival architectural defects, or borderline products and furcation defects, while maintaining periodontal health throughout the balance of the mouth.

- Compromise SPT, designed to slow the progression of disease in patients for whom periodontal corrective therapy is indicated, but cannot be implemented for reasons of health, economics, inadequate oral hygiene, or other considerations, or when recalcitrant defects persist after corrective treatment.

- Post treatment SPT, designated to prevent the recurrence of disease and maintain the periodontal health achieved during therapy.

- This therapy may range from oral hygiene instruction, scaling and other non -surgical approaches to extensive multistage surgical techniques.

\section{Classification of Post-Treatment Patients and Recall Intervals 7,8}

The first year after periodontal therapy is important in terms of the patient in a recall pattern and reinforcing oral hygiene techniques. In addition, it may take several months to accurately evaluate the results of some periodontal surgical procedures.

- Consequently, some areas may have to be retreated because the results may not be optimal.

- $\quad$ Furthermore, the first-year patient often has etiological factors that may have been overlooked and that may be more awardable to treatment at this early stage. For these reasons, the recall intervals for first-year patients should not be longer than 3 months.

- Several categories of maintenance patients and a suggested recall interval for each is shown in the table.

- Supportive periodontal treatment has to be aimed at the regular removal of the sub gingival micro biota and must be supplemented by the patient's effort for optimal supragingival plaque control. A case reported to our department with severe loss of attachment and bone loss. This patient was diagnosed having generalized aggressive periodontitis 5 years back and was treated for the same with full mouth flap surgeries.

Patients susceptible to periodontal disease are at high risk for re-infection and progression of periodontal lesion. So all the patients treated for periodontal disease belong to the risk category by virtue of their past history. Hence an adequate maintenance programme is of utmost importance for a beneficial long-term treatment outcome. ${ }^{9}$

\section{CONCLUSIONS}

It is well recognized that periodontitis is a multifactorial disease. Few of the factors contributing to the onset and progression of periodontal disease can however, be altered by the patient or the clinician, to prevent the recurrence of periodontitis. Some factors can be modulated, i.e. bacterial colonization and smoking. Good control of supragingival plaque is an effective way to prevent disease progression. It has been shown that an effective supragingival oral hygiene may even affect the subgingival micro biota. Successful periodontal therapy with regular SPT can promote periodontal health \& reduce tooth loss. Following active periodontal therapy, an interval should be established for periodic ongoing care.

If the patient was placed on regular SPT, many of the teeth would not have been extracted. The Periodontist's role doesn't end with just completing the surgeries. Frequent recall visits must be scheduled based on the severity of the disease and the prognosis of the case. This would ensure that the treated sites are kept in check against the array of microorganisms and plaque which would otherwise harbour the oral cavity and hinder the oral health. Further research predicting disease activity may include more accurate method for documentation \& assessment by clinical attachment levels, improved imaging technology, microbiological assessment \& enhanced evaluation of GCF component which would permit SPT schedule to be better adapted to the needs of individual patient. 


\section{REFERENCES}

[1] Robert LM, Newman MG, Carranza FA. Periodontal maintenance. In: Newman MG, Takei H, Klokkevold PR, eds. Carranza's Clinical Periodontology. $10^{\text {th }}$ edn. Saunders Elseveir 2007: p. 1194-204.

[2] Gaunt F, Devine M, Pennington M, et al. The costeffectiveness of supportive periodontal care for patients with chronic periodontitis. J Clin Periodontal 2008;35(Suppl 8):67-82.

[3] Ramford SP. Maintenance care for treated periodontitis patients. J Clin Periodontal 1987;14(8):433-7.

[4] Cohen RE, Research, Science and Therapy Committee, American Academy of periodontology. Position paper: parameters on maintenance. J Periodontal 2003;74(9):1395-401.
[5] Axelson P, LIndhe J. The significance of maintenance care in treatment of periodontal disease. J Clin Periodontal 1981;8(4):281-94.

[6] Wilson TG Jr. Compliance. A Review of the literature with possible applications to periodontics. J Periodontal 1987;58(10):706-14

[7] Wilson TG Jr, Glover ME, Schoen J, et al. Compliance with maintenance therapy in private practice. J Periodontal 1984;55(8):468-73.

[8] Renvert S, Persson RG. Supportive periodontal therapy. Periodontology 2000 2004;36(1):179-95.

[9] Caton J, Proye H, Polson A. Maintenance of healed periodontal pockets after single episode of root planing. J Periodontal 1982;53(7):420-4. 